\title{
The 100000 Genomes Project: bringing whole genome sequencing to the NHS
}

The name of one of the authors of this paper (BMJ

2018;361:k1687, doi:10.1136/bmj.k1687) was incorrect. It

should be Andrew Devereau not Devereaux. 